\title{
IHDIP: a controlled randomized trial to assess the security and effectiveness of the incremental hemodialysis in incident patients
}

Javier Deira ${ }^{1 *}$ (D, Miguel A. Suárez² ${ }^{2}$ Francisca López ${ }^{3}$, Emilio García-Cabrera ${ }^{4}$, Antonio Gascón ${ }^{5}$, Eduardo Torregrosa ${ }^{6}$, Giannina E. García ${ }^{7}$, Jorge Huertas ${ }^{8}$, Jose C. de la Flor ${ }^{9}$, Suleya Puello ${ }^{10}$, Jonathan Gómez-Raja ${ }^{11}$, Jesús Grande ${ }^{12}$, José L. Lerma ${ }^{13}$, Carlos Corradino ${ }^{14}$, Carlos Musso ${ }^{14}$, Manuel Ramos ${ }^{15}$, Jesús Martín ${ }^{16}$, Carlo Basile ${ }^{17}$ and Francesco G. Casino ${ }^{17,18}$

\begin{abstract}
Background: Most people who make the transition to renal replacement therapy (RRT) are treated with a fixed dose thrice-weekly hemodialysis réegimen, without considering their residual kidney function (RKF). Recent papers inform us that incremental hemodialysis is associated with preservation of RKF, whenever compared with conventional hemodialysis. The objective of the present controlled randomized trial $(R C T)$ is to determine if start $\mathrm{HD}$ with one sessions per week (1-WK/HD), it is associated with better patient survival and other safety parameters.

Methods/design: IHDIP is a multicenter RCT experimental open trial. It is randomized in a 1:1 ratio and controlled through usual clinical practice, with a low intervention level and non-commercial. It includes 152 incident patients older than 18 years, with a RRF of $\geq 4 \mathrm{ml} / \mathrm{min} / 1.73 \mathrm{~m} 2$, measured by renal clearance of urea (KrU). The intervention group includes 76 patients who will start with incremental HD (1-WK/HD). The control group includes 76 patients who will start with thrice-weekly hemodialysis régimen. The primary outcome is assessing the survival rate, while the secondary outcomes are the morbidity rate, the clinical parameters, the quality of life and the efficiency.
\end{abstract}

Discussion: This study will enable to know the number of sessions a patient should receive when starting HD, depending on his RRF. The potentially important clinical and financial implications of incremental hemodialysis warrant this RCT.

Trial registration: U.S. National Institutes of Health, ClinicalTrials.gov. Number: NCT03239808, completed 13/04/ 2017. Sponsor: Foundation for Training and Research of Health Professionals of Extremadura.

Keywords: Once-weekly haemodialysis, Twice-weekly haemodialysis, Incremental haemodialysis progressive hemodialysis, Randomized clinical trial

\footnotetext{
* Correspondence: deiralorenzo@gmail.com

${ }^{1}$ Hospital San Pedro de Alcantara, Cáceres, Spain

Full list of author information is available at the end of the article
}

C The Author(s). 2019 Open Access This article is distributed under the terms of the Creative Commons Attribution 4.0 International License (http://creativecommons.org/licenses/by/4.0/), which permits unrestricted use, distribution, and reproduction in any medium, provided you give appropriate credit to the original author(s) and the source, provide a link to the Creative Commons license, and indicate if changes were made. The Creative Commons Public Domain Dedication waiver (http://creativecommons.org/publicdomain/zero/1.0/) applies to the data made available in this article, unless otherwise stated. 


\section{Background}

Conventional thrice-weekly HD for 3 to $5 \mathrm{~h}$ in a health center in an outpatient basis is the most used renal replacement therapy (RRT) regimen [1]. However, it has an unacceptable high mortality rate (10-20\% a year). In order to try to improve those results, new regimens have been proposed. They are based on an increase of the HD dose and/or a higher number of sessions [2]. Nevertheless, inconsistent results in terms of clinical benefits with such programs have been shown in recently published randomized and controlled trials, $[3,4]$ together with a lower rate of vascular access success [5] and a lower maintenance of the RRF [6].

The National Kidney Foundation-Kidney Disease Outcomes Quality Initiate (NKD KDOQI 2015) [1] 2015 guidelines allow the reduction in the weekly HD dose for patients with a residual kidney urea clearance $(\mathrm{KrU})$ higher than $3 \mathrm{ml} / \mathrm{min} / 1.73 \mathrm{~m} 2$. In these cases, the renal clearance $(\mathrm{Kr})$ is added to the dialysis clearance $(\mathrm{Kd})$ obtained in 2 sessions per week, thus obtaining the adequate dialysis dose [7, 8]. Surprisingly enough, few centers follow this recommendation when over $50 \%$ of patients start $\mathrm{HD}$ with $\mathrm{KrU}>3 \mathrm{~mL} / \mathrm{min}$ [9].

Authors like Kalantar-Zadeh et al $[9,10]$ in the U.S.A. or Teruel et al [11] in Spain have published their experience with 2 HD sessions per week in incident patients. Through this regime they have shown that the RRF is preserved and the survival rate is similar to the one obtained with the conventional HD. This is due to the fact that the $\mathrm{Kr}$ has much greater clinical weight than $\mathrm{Kd}$, [7] since the RRF contributes to the production of vitamin D and erythropoietine [12, 13], and eliminates the protein-bound uremic toxins that are poorly dialyzed $[13,14]$. In other words, the RRF plays a fundamental role both in the dialysis adequacy and in survival $[15,16]$.

Currently, some authors are questioning the number of HD sessions with which a patient should start the renal replacement therapy (RRT) [7, 17-19]. Progressive HD is an initiation regimen adapted to the patient's RRF. The frequency increases as the diuretic level declines [7, 17-19].

The IHDIP trial [20] aims at determining whether or not starting with one HD session per week reduces mortality in incident patients and its influence in morbidity (hospital admissions), clinical parameters, quality of life and efficiency with regard to the patients who start RRT with the conventional method.

\section{Method and design Trial design}

This is a prospective, multicenter, open clinical trial. It is randomized and controlled through usual clinical practice, based on starting the HD treatment with three sessions per week (control group).

\section{Intervention}

It consists in reducing the frequency or number of sessions per week with which patients start the HD treatment. The experimental group will start with one session/week, then the number of weekly sessions will be increased to two and later to three as per criteria for progression.

Neither drugs nor placebos are used in the IHDIP trial. Complementary procedures in diagnosis or follow-up do not imply any risks for the patients' security, since they are similar to those of the usual clinical practice. This is the reason why it is considered a "low-intensity intervention clinical trial". Likewise, it has been defined as a "Non-commercial clinical trial", since it has been designed directly by researchers without the participation of the pharmaceutical industry.

\section{Ethics and consent to participate}

The study was evaluated and approved (March 28, 2017) by the Ethics and Clinical Research Committee of the San Pedro de Alcántara Hospital in Cáceres, Spain. All participants in the study will receive and sign the informed consent.

\section{Participants}

Hospital and out-patient HD Centres. Only incident patients will be included. Patients admitted due to intercurrent problems will stay in their assigned trial group and will be assessed according to their randomization.

\section{Inclusion criteria}

Patients aged 18 and over with stage 5 chronical kidney disease (CKD) who have chosen HD as treatment modality.

RRF measured by $\mathrm{KrU}$ [21] (see appendix 2) > $4 \mathrm{ml} /$ $\mathrm{min} / 1.73 \mathrm{~m}^{2}$. In general, it is advised not to start HD with a $\mathrm{KrU}>7 \mathrm{ml} / \mathrm{min} / 173 \mathrm{~m}^{2}$.

\section{Exclusion criteria}

Unplanned or urgent initiation of HD treatment. Urgent here means that the urine has not been collected in the 24 hours previous to the first session or that the urine was not collected in the previous 30 days.

Patients who were going through other modalities of RRT.

Associated diseases: Active neoplastic disease, Cardiorenal or hepatorenal syndrome, Active inflammatory disease or Cardiovascular disease defined as heart failure type IV (NYHA), unstable angina or ischemic cardiopathy that has led to a hospital admission in the last 3 months.

\section{Criteria for progression}

The number of weekly sessions of the patients in the experimental group will be increased from one to two sessions in case they meet any of the following criteria:

$\mathrm{KrU}$ [21] level decline (below $4 \mathrm{ml}$ and above $2.5 \mathrm{ml} /$ $\mathrm{min} / 1.73 \mathrm{~m} 2$ ). This decrease must be confirmed in a subsequent sample obtained in the next month. 
Intersessional weekly weight gain which influences an ultrafiltration (UF) rate higher than $13 \mathrm{ml} / \mathrm{kg} /$ hour for a minimum of 3 weeks.

Clinical event that requires non programmed HD sessions (more than one) for its resolution.

Patients with two sessions per week will be changed to the conventional HD method if:

The $\mathrm{KrU}$ [21] level is lower than $2.5 \mathrm{ml} / \mathrm{min} / 1.73 \mathrm{~m}^{2}$. This decrease must be confirmed in a subsequent sample obtained in the next month.

Standard Kt/V is below 2.1 (weekly). This decrease in std. Kt/V must be confirmed in a subsequent sample obtained in the next month.

Intersessional weight gain which influences an ultrafiltration (UF) rate higher $13 \mathrm{ml} / \mathrm{kg} /$ hour for a minimum of 3 sessions.

Clinical event that requires non programmed HD sessions for its resolution.

\section{Participants' schedule}

Recruitment period Eighteen months from the first patient's inclusion. The patients selected as candidates will be registered in the patients' form. If they meet the inclusion criteria and sign the consent form they will start being randomized.

Follow-up period Twenty four months. During this period, biochemical determinations and diagnostic tests will be performed according to the frequency indicated in the visiting schedule. Patients in the experimental group will have the same visits than those in the group of control when they progress to 3 weekly sessions. The work plan is defined in Table 1 and in the Additional file 1.

Removal criteria Any patient will be moved off the trial due to: kidney transplantation, RF recovery, loss of follow-up, program output, or consent withdrawal. In these cases, the final follow-up visit will be carried out and there will be no replacement.

\section{Outcomes}

Primary outcome Survival. Duration of trial: 2 years.

Secondary outcomes Hospital admissions for any reason. Duration of trial: 2 years.

RRF maintenance. Duration of trial: 2 years.

Reduction of glomerular filtration rate (GFR) and tubular function.

Average urine volume and percentage of patients with anuria ( $\leq 200 \mathrm{ml} /$ day in two consecutive measurements).

Adequacy parameters. Duration of trial: 3, 6, 12, 18 months and 2 years.

Anemia control. Patients whose hemoglobin levels are within the therapeutic range (in \%) and the average levels of erythropoietin resistance index (ERI in UI/Kg/ week).

Table 1 Schedule of visits and procedures

\begin{tabular}{|c|c|c|c|c|c|c|}
\hline & Selection Visit & Baseline visit & Monthly visit ${ }^{a}$ & Quarterly visit & Annual visit & Final follow-up visit \\
\hline Inclusion and exclusion criteria & $x$ & & & & & \\
\hline Consent & $x$ & & & & & \\
\hline Demographic data registration & & $x$ & & & & \\
\hline Comorbidity data registration & & $x$ & & & & \\
\hline Primary renal disease diagnosis & & $x$ & & & & \\
\hline Hospital admission & & & $\mathrm{IHD}$ & $x$ & & $x$ \\
\hline Data concerning the technique & & & IHD & $x$ & & $x$ \\
\hline Residual renal function test & & $x$ & $\mathrm{IHD}$ & $x$ & & $x$ \\
\hline Bioimpedance & & $x$ & $\mathrm{IHD}$ & $x$ & & $x$ \\
\hline Acid-base and electrolytic state & & $x$ & $\mathrm{IHD}$ & $x$ & & $x$ \\
\hline Erythropoietic levels & & $x$ & & $x$ & & $x$ \\
\hline Bone-mineral metabolism levels & & $x$ & & $x$ & & $x$ \\
\hline Nutrition- inflammation levels & & $x$ & & $x$ & & $x$ \\
\hline Iron levels & & $x$ & & $x$ & & $x$ \\
\hline KDQOL '36 US Spanish & & $x$ & & $x$ & & $x$ \\
\hline Usual treatment & & $x$ & & $x$ & & $x$ \\
\hline Echocardiogram $^{\mathrm{b}}$ & & $x$ & & & $x$ & $x$ \\
\hline
\end{tabular}

${ }^{a}$ The monthly visit and determinations marked as IHD will only be carried out for patients undergoing incremental HD

${ }^{\mathrm{b}}$ The echocardiogram will only be carried out at the beginning, after 12 months and after 24 months

\& Regarding the data related to the technique, when there are different parameters (e.g. BP weight gain, and so on), only the values obtained in the session when analytical measurements are taken will be registered 
Mineral bone disorder control. Calcium, phosphorus and Parathyroid hormone (PTHi) average levels. Percentage of patients with levels within therapeutic range.

Specific cardiomyopathy control. Duration of trial: 12 and 24 months. Left ventricular ejection fraction (LVEF). Percentage of patients with a left ventricular mass index (LVMI) adjusted to the body surface area $\geq 125 \mathrm{~g} / \mathrm{m}^{2}$, or with pericardial effusion.

Quality of life control. Assessed through the Kidney Disease and Quality of Life Kidney Disease and Quality of Life (KDQOL'36 SF) survey.

Intervention's cost-efficiency ratio: expressed as increased cost per additional quality adjusted life year (QALY) see Additional file 2.

Sample size It was calculated based in the contrast of a null hypothesis $\mathrm{H} 0$ : The rate between the median survival time is not under the limit of no inferiority, through a Log-Rank test for two independent samples (no-inferiority in a function of exponential survival).

Assuming the following parameters: Inclusion period of 18 month, maximum duration of the follow-up period: 24 months, survival median in the conventional HD group: 74 months, time median until censure: 12 months, non-inferiority limit: 4 months, type I error $5 \%$ (significance), and type II error 20\% (capability). We must include 76 patients for the conventional HD group and 76 in incremental HD group, totaling 152 patients in the trial.

Randomization One centralized list has been designed. It includes 152 randomization codes (sample size), and 24 additional ones in case more patients were added. It has two strata: for age ( $\geq$ or $<75$ years old) and for $\mathrm{KrU}$ [21] $\left(\geq\right.$ or $\left.<5,5 \mathrm{ml} / \mathrm{min} / 1.73 \mathrm{~m}^{2}\right)$. The main researcher of each center will formally request the randomization to the Clinical Research Office.

Centralized prescription of the dialysis dose The patient will receive a "centralized prescription" of the dialysis dose, which will be computed quantitatively for each patient. It will be based on the eKt / V necessary according to the $\mathrm{KrU}$ of each patient, to obtain an EKRU of $12-\mathrm{KrU} \mathrm{ml} / \mathrm{min} / 1.73 \mathrm{~m} 2$ on a once-weekly $\mathrm{HD}$ and a stdKt/V of 2.3 weekly volumes for twice -and thriceweekly HD schedules, as published by Casino and Basile [22]. All calculations involving the urea kinetic model (UKM) are based on of the prescription tool [23] and the 'Solute-Solver' software [24] (see Additional file 3). The control group will receive a dose of spkt/V of 1.4 per session, neglecting the residual renal function, as collected by the KDOQI [1].

Note: The KDOQI [1] suggested aiming at $s t d K t / \mathrm{V}=$ $2.3 v / w \mathrm{k}$. for HD schedules other than thrice weekly HD.
But they didn't mention the once-weekly schedule. So, we adopted the recently suggested variable target for EKRU as a guide for once weekly schedule, that seems quite in agreement with our empirical experience.

Variables Data will be obtained from the patient's clinical history. The researchers will fulfill an electronic case report form (eCRF) within the proper periods of time.

Demographic data, clinical data and tests run: Biochemical determinations, diagnostic tests and their frequency are registered in Table 1 . They are the ones that are usually recommended in the guidelines for these patients.

Survival: The follow-up time will be determined in days. It will be defined as the difference in days from the date of the end of the follow-up minus the date of the baseline visit. Events will be counted either as deaths (follow-up of less than 24 months) or as end of the follow-up (24 months).

Hospital admissions: The number of admissions and the admission days will be registered. The following list will be considered as reasons for direct admissions: infections, vascular access, heart failure or ischemic cardiopathology, gastrointestinal bleeding, or other reasons.

RRF maintenance rate: The GFR (in $\mathrm{ml} / \mathrm{min}$ ) will be calculated with the average residual urea and creatinine clearance. The tubular function will be calculated through fractional excretion of phosphorus and uric acid.

Anemia control: The hemoglobin (in g/dl) and the erythropoiesis-stimulating agents (ESA) dose will be measured (in UI).

Mineral bone disorder control: serum phosphorus and calcium levels (in $\mathrm{mg} / \mathrm{dl}$ ), and intact PTH (in $\mathrm{pg} / \mathrm{dl}$ ) will be measured.

Specific cardiomiopathy control: The LVEF (in \%), the LVMI (in $\mathrm{g} / \mathrm{m}^{2}$ ) will be measured, and the presence of pericardial effusion will be assessed.

Quality of life: The items from KDQOL'36 SF survey will be measured.

Intervention's cost-efficiency ratio: During the follow-up, the costs of each patient will be calculated [25]. (see Additional file 2).

\section{Statistical methods \\ Population to analyze}

All patients included in the trial, regardless of their follow-up period. In other words, the population of the trial is population on an intention-to-treat.

Intermediate analysis All the patients' objectives will be analyzed after being followed up for 12 months. In this analysis, the methodology and the variables will be the same to the analysis of results performed at the end of the follow-up (Fig. 1). 
Incident $\mathrm{HD}$ patients $(\mathrm{n}=152)$

Aged $>18$, with stage 5 CKD, planned start and

$\mathrm{KrU}>4 \mathrm{ml} / \mathrm{min} / 1.73 \mathrm{~m} 2$.

All candidates will be registered, even those who are excluded

\begin{tabular}{|c|}
\hline $\begin{array}{l}\text { Centralized randomization } 1: 1 \\
\text { stratified by age }(\geq \text { or }<75 \text { years old) }\end{array}$ \\
\hline 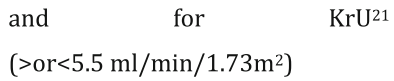 \\
\hline
\end{tabular}

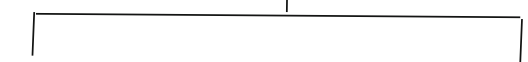

INCREMENTAL HEMODIALYSIS ( $\mathrm{n}=76$ )

start with1 session week

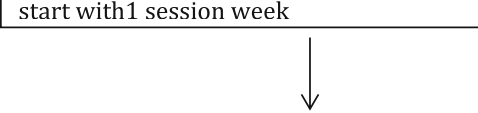

Criteria for progression

- From 1 to 2 session/week:

$\mathrm{KrU}<4 \mathrm{ml} / \mathrm{min} / 1.73 \mathrm{~m} 2$

$\mathrm{UF}$ rate $>13 \mathrm{ml} / \mathrm{kg} / \mathrm{h}$

Clinical event

- From 2 to 3 session/week:

$\mathrm{KrU}<2,5 \mathrm{ml} / \mathrm{min} / 1.73 \mathrm{~m} 2$.

std $\mathrm{Kt} / \mathrm{v}<2.1 \mathrm{v} / \mathrm{w}$. UF rate $>13$

$\mathrm{ml} / \mathrm{kg} / \mathrm{h}$. Clinical event

\section{Removal criteria: kidney transplantation, movement to a different \\ center, loss of follow-up, program output, RF recovery, any exclusion}

criteria, consent withdrawal

Follow-up period: 24 months. Biochemical determinations and

diagnostic tests will be performed according to the frequency indicated

Primary outcome: Survival

Duration of trial: 2 years.

Fig. 1 Schema for IHDIP Trial

Descriptive analysis All the variables registered in the baseline visit will be assessed. Qualitative variables will be expressed in percentage. In order to assess their differences, Chi-square test or statistical Pearson's test will be run, in case the distribution of observed frequencies is not fulfilled. The quantitative variables will be expressed as mean, median, standard deviation and interquartile range. To assess the differences in quantitative variables, the $\mathrm{t}$-Student or the Mann-Whitney test will be performed, in case the normal distribution is not fulfilled. They will have a significance level of $5 \%$ and a capability level of $80 \%$ to meet the outcomes.
Primary outcome Survival assessment: It will be measured through bivariant analysis or Kaplan-Meier test. The differences between mean and median survival in both branches of the trial will be assessed through the logrank test. A multivariant analysis or Cox multivariate regression will be run to assess the actual contribution of the intervention (progressive HD) and/or any other variable that may affect survival.

Secondary outcome Analysis of hospital admissions: In each group, the average value of the number and days of 
admissions will be calculated. The difference among the averages will be assessed through the Student's t- test or Mann-Whitney nonparametric test.

Analysis of the RRF: The evolution of the GFR, the tubular function and the volume of urine $/ 24 \mathrm{~h}$ will be compared following the Wilcoxon test. The RRF's maintenance (volume $\geq 200 \mathrm{ml} /$ day) will be assessed through the Kaplan-Meier procedure. The differences between mean and median will be assessed through the log- rank test.

In order to compare the patients rate (in \%) with a volume of $\leq 200 \mathrm{ml} /$ day, Chi-square test or statistical Pearson's test will be run at the end of the follow-up, according to the distribution of observed frequencies.

Other analytical parameters: In order to compare the percentage of patients with hemoglobin $<10.5 \mathrm{~g} / \mathrm{dl}$, or the levels of calcium, phosphorus and PTH within the therapeutic range (in each branch of the trial), Chi-square test or statistical Pearson's test will be performed if the distribution of frequencies is not fulfilled. The differences among the average levels of ERI, calcium, phosphorus, and intact PTH will be assessed through the Student's t- test or Mann-Whitney nonparametric test.

Functional data: The difference in the LVEF and the LVMI in the quality of life questionnaire items and in the efficiency calculation (in every branch of the trial) will be assessed through the Student's $t$ - test or Mann-Whitney nonparametric test. In order to assess the difference when there is a pericardial effusion, either Chi-square test or statistical Pearson's test will be run if the distribution of frequencies is not fulfilled.

Security controls During the follow-up, and especially in the experimental group, special attention will be paid to volume overload, hyperkalemia and metabolic acidosis, as it is advised in the usual clinical practice. Monthly BIS of patients undergoing incremental HD and quarterly BIS of patients undergoing conventional HD will help calculate the dry weight and dismiss a possible overhydration. This trial will be performed according to the protocol, the GCP guidelines and the applicable national laws and requirements of the countries where the study is being carried out.

Modification of the protocol and access to the final trial dataset Any important modification of the protocol will be updated at ClinicalTrial.gov.

The sponsor or the coordinator/investigators of the trial explicitly commit themselves to publish the results.

\section{Discusion}

Transition of non-dialysis-dependent CKD stage 5 to RRT is a crucial moment, both for the patient and the nephrologist. There must be chosen, among other things: when and how to start the RRT, and the delivered dialysis dose. Even though there is not any controlled study that supports this, there has been a tendency towards an early initiation of RRT [1]. Thus, currently in the USA over $50 \%$ of patients start with a $\mathrm{KrU}>3 \mathrm{~mL} / \mathrm{min} / 1.73 \mathrm{~m}^{2}$, without reducing morbidity and mortality [9].

The aim of starting a progressive dialysis treatment, defined as gradual increase of the dose as the RRF volume decline, is to maintain a continuous total clearance of solutes $(\mathrm{Kr}$ and $\mathrm{Kd})$. This was proposed in the first guidelines for peritoneal dialysis adequacy, [26] and at present it is highly implemented. Thus, in some countries $30 \%$ of patients start with 1 or 2 exchanges/day, or with $\leq 4$ sessions/week of automated PD [27]. This is happening in spite of the limited incremental PD studies, in which there is a low number of patients, who are monocentric and not randomized [27].

Progressive or incremental HD has also become increasingly important over the last years. Performed without economic purposes, it has shown promising results in the RRF maintenance, and the survival is similar to that in conventional HD [9-12]. In fact, the 3.2 guideline in the KDOQI [1] allows reducing the weekly dose in patients with a KrU higher than $3 \mathrm{ml} / \mathrm{min} / 1.73 \mathrm{~m}^{2}$. In these cases, the set objective is to achieve a continuous weekly clearance of 2.3 volumes, expressed in $s t d K t / v$, EKRU of $12-\mathrm{KrU} \mathrm{mL} / \mathrm{min}$, both normalized to a volume of $35 \mathrm{~L}[7,22]$. Such suggestions are based on the strong existing correlation between the RRF and survival [28]; and on their contribution to control the volume and clear protein-bound solutes through tubular secretion $[29,30]$. It should be recalled that these are poorly cleared by current techniques, even when the frequency is increased [31].

However, the published studies on incremental HD are observational, and their results must be regarded with caution. The starting point in most of them was two sessions [9-11]. We could conclude that currently there is not enough evidence that indicates the frequency that HD incident patients with RRF should receive.

Based on previous experiences, [22, 32] and according to some authors, $[19,21]$ in IHDIP "Assessment of the Incremental HemoDialysis security and effectiveness in Incident Patients", we have considered starting with only one weekly session and increase the frequency to two and then to three as the RRF declines. Daring it may seem, but it is more logical gradually to transit from stage $5 \mathrm{NoD}$ to stage $5 \mathrm{HD}$. We hope to get the same survival and complication rates after two years. If this starting regime was corroborated as efficient and safe, it will allow the reduction of sessions to many incident patients. Thus, if one of four HD incident patients in Spain 
would take them gradually, they would avoid going through 76,000 sessions, including the journeys. Besides, the costs would be reduced by more than 21 million Euros annually.

The methodological design was carefully considered. At first an observational cohort study design was chosen, controlling the selection bias through propensity score match. This method must have enough variables in order to avoid biases, which implies that a big control group is needed so that coupled patients can be found. However, that does not eliminate the "residual confounding factors", which are a threat in any observational study. A randomized controlled trial has a minimal bias and provides a higher level of evidence, although it implies noticeable difficulties: lower strength, selection of patients that produces randomization (they may not represent the population on HD), or imbalances between both groups in some key variable. We believe that the sample size calculation and the randomization blocks have minimized such inconveniences and will allow us to find the answer to the raised issue. It does not have data masking for the obvious difficulties that masking the sessions entail.

The IHDIP is likely to be as necessary as other trials such as HEMO, [33] IDEAL [34] or the derivatives from FHN, [3-5] and its results will be as important. But since it is a non-commercial study, there is no funding for including patients. Avoiding underdialysis is an outcome as important as overdialysis. This clinical trial will try to prove whether there is or not a difference between the progressive HD and the thrice-weekly HD schedule for incident patients. Both the potential benefits and the cost savings are obvious reasons for everyone to make such an effort. If you are interested in this issue or you consider the possibility of participating in the study, we will provide you with all the necessary information.

\section{Additional files} Additional file 1: Working plan: here is the work plan to follow in the
study. (DOCX $17 \mathrm{~kb}$ )

Additional file 2: Tools and other calculations: here is a schematic overview on how to obtain blood and urine samples and how to calculate the costs of each patient. (DOCX $16 \mathrm{~kb}$ )

Additional file 3: Urea Kinetic Model: here is shown the main equations of the urea kinetic model used in the study [35-37]. (DOCX 18 kb)

Additional file 4: Institutional Review Board: the full names of all Institutional Review Board (IRBs) which approved the study protocol are cited in this additional file. (DOCX $14 \mathrm{~kb}$ )

\section{Abbreviations}

BIS: Bioimpedance spectrospic; CKD: Chronic kidney disease; eCRF: electronic case report form; ERI: Erythropoietin resistance index; GFR: Glomerular filtration rate; HD: Hemodialysis; IHDIP: Incremental dialysis in incident patients (trial's acronym); Kd: Dialysis clearance; KDOQI: Kidney Disease Outcomes Quality Initiative; KDQOL'36: Kidney Disease and Quality of Life; Kr: Renal clearance; KrU: Renal clearance of urea; LVEF: Left ventricular ejection fraction; LVMI: Left ventricular mass index; RRF: Renal residual function; RRT: Renal replacement therapy; stdKt/v: Standard Kt/v

\section{Acknowledgements}

Doctors J.L. Teruel and F. Maduell for their invaluable collaboration in the trial's methodology and design.

Funding

Currently, the project is being supported by Fundación Liberbank with $€ 10,000$ net.

Availability of data and materials

Not Applicable.

\section{Protocol}

The complete Study Protocol and Statistical Analysis Plan([PDF] August 2 2017) is available at https://clinicaltrials.gov/ct2/show/NCT03239808

\section{Authors' contributions}

All authors have actively contributed in the trial's design, methodology and writing. More specifically, JD, MAS, CB and FGC designed and critically reviewed the trial. EGC, JGR, AG, FL, ET, GEG, JH, JCF, SP, JG, JLL, CC, CM, MR, and $J M$, have provided substantial contributions to conception and design. All authors read and approved the final manuscript.

\section{Ethics approval and consent to participate}

The study was evaluated and approved (March 28, 2017) by the institutional review board (IRB) of the San Pedro de Alcántara Hospital in Cáceres, Spain. According to the national regulation of clinical trials, the main IRB enables other centers in Spain to carry out the study, providing umbrella coverage for the Spanish centers. However other IRB have evaluated the protocol, the full names of all IRBs which approved the study protocol are listed in an Additional file 4. All participants in the study will be informed about their potential participation and asked to sign the informed consent, if agree to participate. The trial was endorsed by the Spanish Society of Nephrology.

Consent for publication

"Not Applicable".

\section{Competing interests}

The authors declare that they have no competing interests.

\section{Publisher's Note}

Springer Nature remains neutral with regard to jurisdictional claims in published maps and institutional affiliations.

\section{Author details}

${ }^{1}$ Hospital San Pedro de Alcantara, Cáceres, Spain. ${ }^{2}$ Hospital Virgen Del Puerto, Plasencia, Spain. ${ }^{3}$ Hospital Costa del Sol, Marbella, Spain. ${ }^{4}$ Delos Clinical Research Organization, Sevilla, Spain. ${ }^{5}$ Hospital Obispo Polanco, Teruel, Spain. ${ }^{6}$ Hospital de Manises, Valencia, Spain. ${ }^{7}$ Hospital Arquitecto Marcide, Ferrol, Spain. ${ }^{8}$ Hospital de Especialidades de las Fuerzas Armadas, Quito, Ecuador. ${ }^{9}$ Hospital Central de la Defensa Gómez Ulla, Madrid, Spain. ${ }^{10}$ Hospital Clínico Universitario, Santiago de Compostela, Spain. ${ }^{11}$ FundeSalud, Mérida, Spain.

${ }^{12}$ Hospital Virgen de la Concha, Zamora, Spain. ${ }^{13}$ Complejo Asistencial Universitario, Salamanca, Spain. ${ }^{14}$ Hospital Durand de Buenos Aires, Buenos Aires, Argentina. ${ }^{15}$ Hospital de Jerez, Cádiz, Spain. ${ }^{16}$ Hospital Nuestra Sra. de Sonsoles, Ávila, Spain. ${ }^{17}$ Clinical Research Branch, Division of Nephrology, Miulli General Hospital, Acquaviva delle Fonti, Bari, Italy. ${ }^{18}$ Dialysis Centre SM2, Potenza, Italy.

Received: 24 January 2018 Accepted: 17 December 2018 Published online: 09 January 2019

\section{References}

1. National Kidney Foundation. KDOQI clinical practice guideline for hemodialysis adequacy: 2015 update. Am J Kidney Dis. 2015;66(5):884-930.

2. Chan CT, Covic A, Craig JC, et al. Novel techniques and innovation in blood purification: a clinical update from kidney disease: improving global outcomes. Kidney Int. 2013;83(3):359-71. 
3. Chertow GM, Levin NW, Beck GJ, et al. Frequent Hemodialysis Network (FHN) Trials Group. Long-term effects of frequent in-center hemodialysis. J Am Soc Nephrol. 2016;27(6):1830-6.

4. Rocco MV, Daugirdas JT, Greene T, et al. Long-term effects of frequent nocturnal hemodialysis on mortality: the frequent hemodialysis network (FHN) nocturnal trial. Am J Kidney Dis. 2015;66(3):459-68.

5. Suri RS, Larive B, Sherer $S$, et al. Frequent hemodialysis network trial group. Risk of vascular access complications with frequent hemodialysis. J Am Soc Nephrol. 2013;24(3):498-505.

6. Daugirdas JT, Greene T, Rocco MV, et al. FHN trial group. Effect of frequent hemodialysis on residual kidney function. Kidney Int. 2013;83(5):949-58.

7. Casino FG, Basile C. The variable target model: a paradigm shift in the incremental haemodialysis prescription. Nephrol Dial Transplant. 2017;32(1): 182-90.

8. Termorshuizen F, Dekker FW, van Manen JG, et al. Relative contribution of residual renal function and different measures of adequacy to survival in hemodialysis patients: an analysis of the Netherlands cooperative study on the adequacy of Dialysis (NECOSAD)-2. J Am Soc Nephrol. 2004;15(4):1061-70.

9. Obi Y, Streja E, Rhee CM, et al. Incremental hemodialysis, residual kidney function, and mortality risk in incident Dialysis patients: a cohort study. Am J Kidney Dis. 2016;68(2):256-65

10. Zhang $M$, Wang $M$, Li H, et al. Association of initial twice-weekly hemodialysis treatment with preservation of residual kidney function in ESRD patients. Am J Nephrol. 2014;40(2):140-50.

11. Fernández $M$, Teruel JL. Incremental hemodialysis schedule at the start of renal replacement therapy. Nefrologia. 2017;37(1):1-4.

12. Vilar $\mathrm{E}$, Wellsted $\mathrm{D}$, Chandna SM, et al. Residual renal function improves outcome in incremental haemodialysis despite reduced dialysis dose. Nephrol Dial Transplant. 2009;24(8):2502-10.

13. Mathew AT, Fishbane S, Obi Y, Kalantar-Zadeh K. Preservation of residual kidney function in hemodialysis patients: reviving an old concept. Kidney Int. 2016;90(2):262-71.

14. Patel N, Hu SL. Preserving residual renal function in dialysis: what we know. Semin Dial. 2015;28(3):250-8.

15. Shafi T, Jaar BG, Plantinga LC, et al. Association of residual urine output with mortality, quality of life, and inflammation in incident hemodialysis patients: the choices for healthy outcomes in caring for end-stage renal disease (CHOICE) study. Am J Kidney Dis. 2010;56(2):348-58.

16. van der Wal WM, Noordzij M, Dekker FW, et al. Netherlands Cooperative Study on the Adequacy of Dialysis Study Group (NECOSAD). Full loss of residual renal function causes higher mortality in dialysis patients; findings from a marginal structural model. Nephrol Dial Transplant. 2011;26(9):2978-83.

17. Libetta C, Nissani P, Dal Canton A. Progressive Hemodialysis: Is It The Future? Semin Dial. 2016;29(3):179-83.

18. Toth-Manikowski SM, Shafi T. Hemodialysis prescription for incident patients: twice seems Nice, but is it incremental. Am J Kidney Dis. 2016;68(2):180-3.

19. Wong J, Vilar E, Davenport A, et al. Incremental haemodialysis. Nephrol Dial Transplant. 2015;30(10):1639-48.

20. In: Hemodiálisis incremental en pacientes incidentes (IHDIP). U.S. National Institutes of Health, ClinicalTrials.gov. https://clinicaltrials.gov/ct2/show/ NCT03239808. Accessed 13 Apr 2018.

21. Obi Y, Kalantar-Zadeh K, Streja E, Daugirdas JT. Prediction equation for calculating residual kidney urea clearance using urine collections for different hemodialysis treatment frequencies and interdialytic intervals. Nephrol Dial Transplant. 2018:33(3):530-9.

22. Casino FG, Basile C. How to set the stage for a full-fledged clinical trial testing 'incremental haemodialysis'. Nephrol Dial Transplant. 2018;33(7): 1103-9

23. Casino FG, Basile C. A user-friendly tool for incremental haemodialysis prescription. Nephrol Dial Transplant. 2018;33(6):1074-5.

24. Daugirdas JT, Depner TA, Greene T, et al. Solute-solver: a web-based tool for modeling urea kinetics for a broad range of hemodialysis schedules in multiple patients. Am J Kidney Dis. 2009:54:798-809.

25. Parra E, Arenas MD, Alonso M, et al. Grupo de Gestión de la Calidad de la Sociedad Española de Nefrología. Multicentre study of haemodialysis costs Nefrologia. 2011;31(3):299-307.

26. National Kidney Foundation. NKF-DOQI clinical practice guidelines for peritoneal dialysis adequacy. Am J Kidney Dis. 1997;30(3 Suppl 2):S67-136.

27. Neri L, Viglino G, Marinangeli G, et al. Peritoneal Dialysis study Group of Italian Society of nephrology. Incremental start to PD as experienced in Italy: results of censuses carried out from 2005 to 2014. J Nephrol. 2017;30(4): 593-9.

28. Termorshuizen F, Dekker FW, van Manen JG, et al. NECOSAD Study Group. Relative contribution of residual renal function and different measures of adequacy to survival in hemodialysis patients: an analysis of the Netherlands cooperative study on the adequacy of Dialysis (NECOSAD)-2. J Am Soc Nephrol. 2004;15(4):1061-70.

29. Meyer TW, Peattie JW, Miller JD, et al. Increasing the clearance of proteinbound solutes by addition of a sorbent to the dialysate. J Am Soc Nephrol. 2007;18(3):868-74

30. Sirich TL, Funk BA, Plummer NS, et al. Prominent accumulation in hemodialysis patients of solutes normally cleared by tubular secretion. J Am Soc Nephrol. 2014;25(3):615-22.

31. Sirich $\mathrm{TL}$, Fong $\mathrm{K}$, Larive $\mathrm{B}$, et al. Limited reduction in uremic solute concentrations with increased dialysis frequency and time in the frequent hemodialysis network daily trial. Kidney Int. 2017;91:1186-92.

32. González-Sanchidrián S, Deira J, Suárez MA. Progressive hemodialysis: is it the future, or the present? Semin Dial. 2017;30(1):80.

33. Eknoyan G, Beck GJ, Cheung AK, et al. Effect of dialysis dose and membrane flux in maintenance hemodialysis. N Engl J Med. 2002;347(25):2010-9.

34. Cooper BA, Branley $P$, Bulfone L, et al. A randomized, controlled trial of early versus late initiation of dialysis. N Engl J Med. 2010;363(7):609-19.

35. Daugirdas JT. Estimating time-averaged serum urea nitrogen concentration during various urine collection periods: a prediction equation for thrice weekly and biweekly dialysis schedules. Semin Dial. 2016;29:507-9.

36. Daugirdas JT, Leypoldt KJ, Akonur A, Greene T, Depner TA. The FHN trial group. Improved equation for estimating single-pool Kt/N at higher dialysis frequencies. Nephrol Dial Transplant. 2013;28:2156-60.

37. Daugirdas JT, Smye SW. Effect of a two compartment distribution on apparent urea distribution volume. Kidney Int. 1997:51:1270-723.

\section{Ready to submit your research? Choose BMC and benefit from:}

- fast, convenient online submission

- thorough peer review by experienced researchers in your field

- rapid publication on acceptance

- support for research data, including large and complex data types

- gold Open Access which fosters wider collaboration and increased citations

- maximum visibility for your research: over $100 \mathrm{M}$ website views per year

At $\mathrm{BMC}$, research is always in progress.

Learn more biomedcentral.com/submission 